\title{
Almost global existence for a fractional Schrödinger equation on spheres and tori
}

\author{
Dario Bambusi and Yannick Sire
}

Communicated by Y. Charles Li, received January 14, 2013.

\begin{abstract}
We study the time of existence of the solutions of the following Schrödinger equation

$$
i \psi_{t}=(-\Delta)^{s} \psi+f\left(|\psi|^{2}\right) \psi, \quad x \in \mathbb{S}^{d}, \text { or } x \in \mathbb{T}^{d}
$$

where $(-\Delta)^{s}$ stands for the spectrally defined fractional Laplacian with $s>$ $1 / 2$ and $f$ a smooth function. We prove an almost global existence result for almost all $s>1 / 2$.
\end{abstract}

\section{Contents}

1. Introduction and result

\section{Introduction and result}

We consider the equation

$$
i \psi_{t}=(-\Delta)^{s} \psi+f\left(|\psi|^{2}\right) \psi, x \in \mathbb{S}^{d} \text { or } x \in \mathbb{T}^{d},
$$

where $(-\Delta)^{s}$ is the spectrally defined fractional Laplacian, i.e. the $s$-th power of the Laplace-Beltrami operator $-\Delta$ and $f$ a function of class $C^{\infty}$ in a neighborhood of 0 .

We will study such an equation in high regularity Sobolev spaces. We denote by $H^{r}$ the Sobolev space of the $L^{2}$ functions which admit $r$ weak derivatives which are square integrable. We will endow it with the norm

$$
\|\psi\|_{H^{r}}^{2}:=\int\left[|\psi(x)|^{2}+\bar{\psi}(x)(-\Delta)^{r} \psi(x)\right] d x .
$$

1991 Mathematics Subject Classification. 35.

Key words and phrases. Almost global existence, fractional Schrödinger equation. 
Our main result is the following theorem.

THEOREM 1.1. Fix $K \geq 1$, then there exists a set $\mathcal{S} \subset(1 / 2,+\infty)$, having zero measure, such that, for any $s \in(1 / 2,+\infty)-\mathcal{S}$ there exists a positive $r_{K}$ and for any $r>r_{K}$ there exist $\epsilon_{r, K}, T_{r, K}$ with the following property: if the initial datum $\psi_{0}$ fulfills

$$
\epsilon:=\left\|\psi_{0}\right\|_{H^{r}}<\epsilon_{r, K}
$$

then one has

$$
\|\psi(t)\|_{H^{r}}<2 \epsilon, \quad \forall|t| \leq \frac{T_{r, K}}{\epsilon^{K}} .
$$

We remark that our method is unable to deal with $s \in(0,1 / 2)$, due to the fact that the growth of the frequencies is too slow and thus the differences between couple of frequencies can accumulate on open sets.

The theorem is an application of Theorem 4.3 (and Proposition 4.1) of [Bam08], which in turn is based on the Birkhoff normal form theory developed in [Bam03, BG06, BDGS07, Gré07, Bam08]. Some care is needed in order to apply such a theory, because in our case one of the frequencies vanishes and thus, a priori, the normal form does not allow to control the motion of the corresponding mode. Furthermore, one has to check that the frequencies fulfill the strong nonresonance condition introduced in those papers. The first problem is solved exploiting the Gauge invariance of the equation (more or less as in [BS07]), the second one by a variant of the method used in [Bam03] for the wave equation.

We recall that such a theory has been recently extended by Delort to some quasilinear equations [Del12, Del11], however we did not investigate the applicability of his method to the present case.

Concerning existence of the dynamics of the fractional Schrödinger equation, local existence of smooth solution is trivial. On the contrary, as far as the dimension is larger then 1, very little is known on the time of existence of solutions, indeed, to the knowledge of the authors, the only existing results are those of $[\mathbf{G W}]$ in $\mathbb{R}^{d}$, where dispersion is exploited in order to prove global-wellposedness and scattering for small data. On the contrary nothing is known on compact manifold, where such a mechanism clearly fails.

\section{Proof of Theorem 1.1}

To be determined we focus on the case of the sphere which is slightly more difficult. The case of tori is almost identical.

We expand $\psi$ in spherical harmonics

$$
\psi=\sum_{j, k} \xi_{j k} Y_{j k}(x)
$$

and

$$
\bar{\psi}=\sum_{j, k} \eta_{j k} Y_{j k}(x)
$$

where $Y_{j k}$ are the spherical harmonics.

The Hamiltonian writes

$$
H(\xi, \eta)=\sum_{j \geq 0} \omega_{j} \sum_{k} \xi_{j k} \eta_{j k}+H_{p}(\xi, \eta)
$$


where $\omega_{j}=(j(j+d-1))^{s}$ and $H_{p}$ has a zero of order 3 at the origin. Then eq. (1) is equivalent to the Hamilton equations

$$
\dot{\xi}_{j k}=-i \frac{\partial H}{\partial \eta_{j k}}, \quad \dot{\eta}_{j k}=i \frac{\partial H}{\partial \xi_{j k}} .
$$

We will also use the Poisson brackets of two functions $F, G$ on the phase space, which are defined by

$$
\{F ; G\}:=i \sum_{j k}\left[\frac{\partial F}{\partial \eta_{j k}} \frac{\partial G}{\partial \xi_{j k}}-\frac{\partial F}{\partial \xi_{j k}} \frac{\partial G}{\partial \eta_{j k}}\right] .
$$

The theory developed in [DS04, Bam03, BG06, BDGS07, Gré07, Bam08] applies to Hamiltonians of the form (5) in which the nonlinear part belongs to a suitable class (called functions with localized coefficients in [Bam08]). It was proved in [Bam08] that in our case the nonlinearity belongs to such a class, so we refer to that paper for the proof and the precise definition of such a property.

We come to the nonresonance property of the frequencies.

Definition 2.1. Fix $K \geq 3$, then the frequencies $\left(\omega_{1}, \ldots, \omega_{\infty}\right)$ are said to fulfill the property $(K$-NR) if there exist $\gamma>0$, and $\alpha \in \mathbb{R}$ such that for any $N$ large enough one has

$$
\left|\sum_{j \geq 1} \omega_{j} L_{j}\right| \geq \frac{\gamma}{N^{\alpha}},
$$

for any $L \in \mathbb{Z}^{\infty}$, fulfilling $0 \neq|L|:=\sum_{j}\left|L_{j}\right| \leq K+2, \sum_{j>N}\left|L_{j}\right| \leq 2$.

We are going to prove that for almost all $s$ in the considered interval such a property is fulfilled.

TheOREM 2.1. There exists a zero measure set $\mathcal{S} \subset(1 / 2,+\infty)$ such that if $s \in(1 / 2,+\infty)-\mathcal{S}$ then the frequencies

fulfill the property $(K-N R)$

$$
\left(\omega_{1}, \ldots, \omega_{\infty}, \ldots\right)
$$

The proof of such a theorem is a straightforward generalization of the proof of Theorem 4.4 of [Bam08]. The only difference is given by the estimate from below of the determinant of the matrix formed by the vectors of the derivatives of the frequencies with respect to $s$. Such an estimate is done explicitly in the following Lemma 2.2.

We also remark that the condition $s>1 / 2$ is needed in order to pass from Lemma 6.8 of [Bam08] to Lemma 6.9 of the same paper, namely for passing from the estimate of linear combinations of frequencies with index smaller then a fixed $K$ to linear combinations involving also a couple of arbitrary large indexes (as in the second Melnikov condition of KAM theory).

Lemma 2.2. For any $\kappa \leq K$, consider $\kappa$ indexes $1 \leq j_{1} \leq \cdot \leq j_{\kappa} \leq K$; consider the matrix $D$ given by

$$
\left(\begin{array}{ccc}
\omega_{j_{1}} & \cdots & \omega_{j_{\kappa}} \\
d \omega_{j_{1}} / d s & \cdots & d \omega_{j_{\kappa}} / d s \\
\vdots & \vdots & \vdots \\
d^{\kappa-1} \omega_{j_{1}} / d s^{\kappa-1} & \cdots & d^{\kappa-1} \omega_{j_{\kappa}} / d s^{\kappa-1}
\end{array}\right)
$$


Denote by $\mathcal{D}$ its determinant. Then there exists $C>0$ s.t. the following estimate holds

$$
|\mathcal{D}| \geq \frac{C}{K^{\kappa^{2}}}
$$

Proof. Denote

$$
\lambda_{j}=j(j+d-1)
$$

then one has

$$
\frac{d^{k} \omega_{j}}{d s^{k}}=\left(\ln \lambda_{j}\right)^{k} \omega_{j}
$$

Therefore

$$
\begin{gathered}
\mathcal{D}=\omega_{j_{1}} \cdots \omega_{j_{\kappa}}\left|\begin{array}{ccc}
1 & \cdots & 1 \\
x_{j_{1}} & \cdots & x_{j_{\kappa}} \\
\vdots & \vdots & \vdots \\
x_{j_{1}}^{\kappa-1} & \cdots & x_{j_{\kappa}}^{\kappa-1}
\end{array}\right| \\
=\omega_{j_{1}} \cdots \omega_{j_{\kappa}} \prod_{1 \leq l<k \leq \kappa}\left(x_{j_{k}}-x_{j_{l}}\right)=\omega_{j_{1}} \cdots \omega_{j_{\kappa}} \prod_{1 \leq l<k \leq \kappa} \ln \frac{\lambda_{j_{k}}}{\lambda_{j_{l}}}
\end{gathered}
$$

where $x_{j}:=\ln \lambda_{j}$. To fix ideas take $j_{k}>j_{l}$, and let $\delta=\lambda_{j_{k}}-\lambda_{j_{l}}=\left(j_{k}-j_{l}\right)\left(j_{k}+\right.$ $\left.j_{l}+d-1\right)>d$. Then

Thus

$$
\ln \frac{\lambda_{j_{k}}}{\lambda_{j_{l}}}=\ln \left(1+\frac{\delta}{\lambda_{j_{l}}}\right) \geq \ln \left(1+\frac{d}{\lambda_{j_{l}}}\right) \geq \frac{C}{j_{l}^{2}} .
$$

$$
\prod_{1 \leq \ell<k \leq \kappa} \ln \frac{\lambda_{j_{k}}}{\lambda_{j_{l}}} \geq \prod \frac{C}{K^{2}} \geq \frac{C}{K^{2 \kappa^{2}}} .
$$

Since $\omega_{j} \geq 1$ for all $j$ the thesis follows.

Thus, proceeding as in [Bam08] one gets that the frequencies fulfill the nonresonance condition and Theorem 4.15 of [Bam08] applies. Such a theorem ensures that, defining

$$
I_{j}:=\sum_{l} \xi_{j l} \eta_{j l} \equiv \sum_{l}\left|\xi_{j l}\right|^{2}
$$

the following holds.

THEOREM 2.3. (Theorem 4.15 of [Bam08])Fix $K \geq 1$, then there exists a finite $r_{K}$ a neighborhood $\mathcal{U}_{r_{K}}^{(K)}$ of the origin in $H^{r_{K}}$ and a canonical transformation $\mathcal{T}_{K}: \mathcal{U}_{r_{K}}^{(K)} \rightarrow H^{r_{K}}$ which puts the system in normal form up to order $K+3$, namely s.t.

$$
H^{(K)}:=H \circ \mathcal{T}_{K}=H_{0}+Z^{(K)}+\mathcal{R}^{(K)}
$$

where $Z^{(K)}$ and $\mathcal{R}^{(K)}$ have smooth vector field

(i) $Z^{(K)}$ is a polynomial of degree $K+2$ which Poisson commutes with $I_{j}$ for all $j \neq 0$ (but not necessarily with $I_{0}$;

(ii) $\mathcal{R}^{(K)}$ has a small vector field, i.e.

$$
\left\|X_{\mathcal{R}^{(K)}}(\psi)\right\|_{H^{r_{K}}} \leq C\|\psi\|_{H^{r_{K}}}^{K+2}, \quad \forall \psi \in \mathcal{U}_{r_{K}}^{(K)} ;
$$


(iii) one has

$$
\left\|\psi-\mathcal{T}_{K}(\psi)\right\|_{H^{r_{K}}} \leq C\|\psi\|_{H^{r_{K}}}^{2}, \quad \forall \psi \in \mathcal{U}_{r_{K}}^{(K)} .
$$

An inequality identical to (12) is fulfilled by the inverse transformation $\mathcal{T}_{K}^{-1}$.

(iv) For any $r \geq r_{K}$ there exists a subset $\mathcal{U}_{r}^{(K)} \subset \mathcal{U}_{r_{K}}^{(K)}$ open in $H^{r}$ such that the restriction of the canonical transformation to $\mathcal{U}_{r}^{(K)}$ is analytic also as a map from $H^{r} \rightarrow H^{r}$ and the inequalities (11) and (12) hold with $r$ in place of $r_{K}$.

This theorem however is not enough to control the solution since as emphasized at point (i), due to the fact that the zero mode has zero frequency, $I_{0} \equiv \xi_{0} \eta_{0}$ does not commute with $Z^{(K)}$ and thus its modulus can a priori grow in an unbounded way. However, as we are going to show in a while, this cannot happen due to the Gauge invariance.

REMARK 2.4. Due to Gauge invariance, the $L^{2}$ norm is preserved for the original nonlinear dynamics and since $-\Delta$ is self-adjoint on $L^{2}$ one has

$$
\Gamma(\xi, \eta):=\int_{\mathbb{S}^{d}} \psi \bar{\psi} d x=\sum_{j k} \eta_{j k} \xi_{j k}=\sum_{j, k}\left|\xi_{j k}\right|^{2} .
$$

REMARK 2.5. Expanding $H_{p}$ is Taylor series one has

$$
H_{p}(\xi, \eta)=\sum_{J, L} H_{J L} \eta^{L} \xi^{J}, \quad \eta^{L}:=\prod_{j k} \eta_{j k}^{L_{j k}}
$$

and similarly for $\xi^{J}$. Due to Gauge invariance $H_{J L} \neq 0$ implies

$$
\left\{\Gamma, \xi^{L} \eta^{J}\right\}=i \sum_{j k}\left(L_{j k}-J_{j k}\right) \xi^{L} \eta^{J}=0
$$

which, in turn implies

$$
\sum_{j k}\left(L_{j k}-J_{j k}\right)=0
$$

End of the proof of Theorem 1.1. The Gauge invariance is conserved after the Birkhoff normal form transformation (see e.g. [BS07]). It follows that any monomial $\xi^{L} \eta^{J}$ which is present in the normal form $Z^{(K)}$ fulfills the property (13). However, if a monomial is present in the normal form it must also commute with all the $I_{j}, j \neq 0$. It follows that the indexes must fulfill

$$
\sum_{l}\left(L_{j k}-J_{j k}\right)=0, \quad \forall j \neq 0,
$$

which together with (13) implies $L_{0}-J_{0}=0$, which in turn implies that $\left\{Z^{(K)}, I_{0}\right\}=$ 0. Then Theorem 1.1 follows exactly in the same way in which Proposition 4.1 of [Bam08] follows from Theorem 4.3 of that paper.

\section{References}

[Bam03] Dario Bambusi. Birkhoff normal form for some nonlinear PDEs. Comm. Math. Phys., 234(2):253-285, 2003.

[Bam08] D. Bambusi. A Birkhoff normal form theorem for some semilinear PDEs. In Hamiltonian dynamical systems and applications, NATO Sci. Peace Secur. Ser. B Phys. Biophys., pages 213-247. Springer, Dordrecht, 2008. 
[BDGS07] D. Bambusi, J.-M. Delort, B. Grébert, and J. Szeftel. Almost global existence for Hamiltonian semilinear Klein-Gordon equations with small Cauchy data on Zoll manifolds. Comm. Pure Appl. Math., 60(11):1665-1690, 2007.

[BG06] D. Bambusi and B. Grébert. Birkhoff normal form for partial differential equations with tame modulus. Duke Math. J., 135(3):507-567, 2006.

[BS07] Dario Bambusi and Andrea Sacchetti. Exponential times in the one-dimensional GrossPitaevskii equation with multiple well potential. Comm. Math. Phys., 275(1):1-36, 2007.

[Del11] Jean-Marc Delort. Quasi-linear perturbations of Hamiltonian Klein-Gordon equations on spheres. November 2011. http://hal.archives-ouvertes.fr/hal-00643474/PDF/article.pdf

[Del12] J.-M. Delort. A quasi-linear Birkhoff normal forms method. Application to the quasilinear Klein-Gordon equation on $\mathbb{S}^{1}$. Astérisque, (341):vi+113, 2012.

[DS04] J.-M. Delort and J. Szeftel. Long-time existence for small data nonlinear Klein-Gordon equations on tori and spheres. Int. Math. Res. Not., (37):1897-1966, 2004.

[Gré07] Benoît Grébert. Birkhoff normal form and Hamiltonian PDEs. In Partial differential equations and applications, volume 15 of Sémin. Congr., pages 1-46. Soc. Math. France, Paris, 2007.

[GW] Z. Guo and Y. Wang. Improved Strichartz estimates for a class of dispersive equations in the radial case and their applications to nonlinear schrdinger and wave equation. Preprint.

Universitì degli studi di Milano, Dipartimento di Matematica, Via Saldini 50, 20133 Milano, ITALia

E-mail address: dario.bambusi@unimi.it

Université Aix-Marseille Centre de Mathématique et Informatique Laboratoire D'Analyse, Topologie, Probabilité (LATP) Technopole de Chateau-Gombert, 9, rue F. Joliot Curie, 13453 Marseille Cedex 13, France

E-mail address: sire@cmi.univ-mrs.fr 